\title{
Tuberculosis treatment outcome in the European Union and European Economic Area: an analysis of surveillance data from 2002-2011
}

B Karo ${ }^{12}$, B Hauer ${ }^{1}$, V Hollo $^{3}$, MJ van der Werf ${ }^{3}$, L Fiebig $^{1}$, W Haas ${ }^{1}$

1. Department for Infectious Disease Epidemiology, Robert Koch Institute (RKI), Berlin, Germany

2. PhD Programme Epidemiology, Braunschweig-Hannover, Germany

3. European Centre for Disease Prevention and Control (ECDC), Stockholm, Sweden

Correspondence: Basel Karo (karob@rki.de)

Citation style for this article:

Karo B, Hauer B, Hollo V, van der Werf M, Fiebig L, Haas W. Tuberculosis treatment outcome in the European Union and European Economic Area: an analysis of surveillance data from 2002D2011. Euro Surveill. 2015;20(49):pii=30087. DOI: http://dx.doi.org/10.2807/1560-7917.ES.2015.20.49.30087

Article submitted on 03 February 2015 / accepted on 24 July 2015 / published on 10 December 2015

Monitoring the treatment outcome (TO) of tuberculosis (TB) is essential to evaluate the effectiveness of the intervention and to identify potential barriers for TB control. The global target is to reach a treatment success rate (TSR) of at least $85 \%$. We aimed to assess the TB TO in the European Union and European Economic Area (EU/EEA) between 2002 and 2011, and to identify factors associated with unsuccessful treatment. Only 18 countries reported information on TO for the whole observation period accounting for 250,854 new culture-confirmed pulmonary TB cases. The $85 \%$ target of TSR was not reached in any year between 2002 and 2011 and was on average $78 \%$. The TSR for multidrug-resistant (MDR)-TB cases at 24-month follow-up was $49 \%$. In the multivariable regression model, unsuccessful treatment was significantly associated with increasing age (odds ratio $(O R)=1.02$ per a one-year increase, $95 \%$ confidence interval $(\mathrm{Cl}): 1.02-$ 1.02), MDR-TB (OR=8.7, 95\% Cl: 5.09-14.97), male sex $(O R=1.40,95 \% \mathrm{Cl}: 1.28-1.52)$, and foreign origin $(\mathrm{OR}=1.32,95 \% \mathrm{Cl}: 1.03-1.70)$. The data highlight that special efforts are required for patients with MDR-TB and the elderly aged $\geq 65$ years, who have particularly low TSR. To allow for valid monitoring at EU level all countries should aim to report TO for all TB cases.

\section{Introduction}

In 1991, the 44th World Health Assembly set targets to detect at least $70 \%$ of new tuberculosis (TB) cases and to cure at least $85 \%$ of those detected [1]. The Stop TB Partnership developed the Global Plan to Stop TB 2006-2015 to achieve these targets set for 2015 within the context of the Millennium Development Goals [2]. Monitoring the outcome of TB treatment is essential to evaluate the effectiveness of the intervention and to identify the potential barriers for TB control.
In Europe, a Working Group of the World Health Organization (WHO) and the International Union against Tuberculosis and Lung Disease (IUATLD) published recommendations for uniform reporting by TB surveillance and cohort analysis of treatment outcome (TO) across Europe $[3,4]$. A minimal set of six exclusive categories of TO was recommended as standard: cured, completed, failed, died, interrupted (defaulted) and transferred out. Furthermore, analysis of TO should be separate for new and retreatment cases [4]. In 2008, the European Centre for Disease Prevention and Control (ECDC) published the Framework Action Plan to fight Tuberculosis in the European Union following the WHO/IUATLD recommendations; including a core indicator of $85 \%$ treatment success rate for new pulmonary culture-confirmed TB cases and $70 \%$ for new pulmonary culture-confirmed multidrug-resistant (MDR) TB cases [5].

In 2013, in the European Union and European Economic Area (EU/EEA), the TB notification rate was 12.7 per 100,000 population [6]. Notification rates were heterogeneous: five countries had incidence rates $\geq 20$ and 24 countries had incidence rates $<20$ cases per 100,000 population in 2013) [6]. In the majority of countries the trend in case notification rate showed a sustained decline during the period 2009-2013. In 2013, the overall treatment success rate was $73.5 \%$.

In this study, we aimed to assess the TB TO in the EU/ EEA and to identify factors associated with unsuccessful treatment applying the WHO/IUATLD recommendations for the EU/EEA for cohort analysis over a 10-year observation period. 


\section{FIGURE 1}

Algorithm for inclusion of cases in the analysis on treatment outcome of new culture-confirmed pulmonary tuberculosis, EU/EEA, 2002-2011 $(n=589,688)$ Algorithm for inclusion of cases in the analysis on treatment outcome of new culture-confirmed pulmonary tuberculosis, EU/ EEA, 2002-2011 $(n=589,688)$

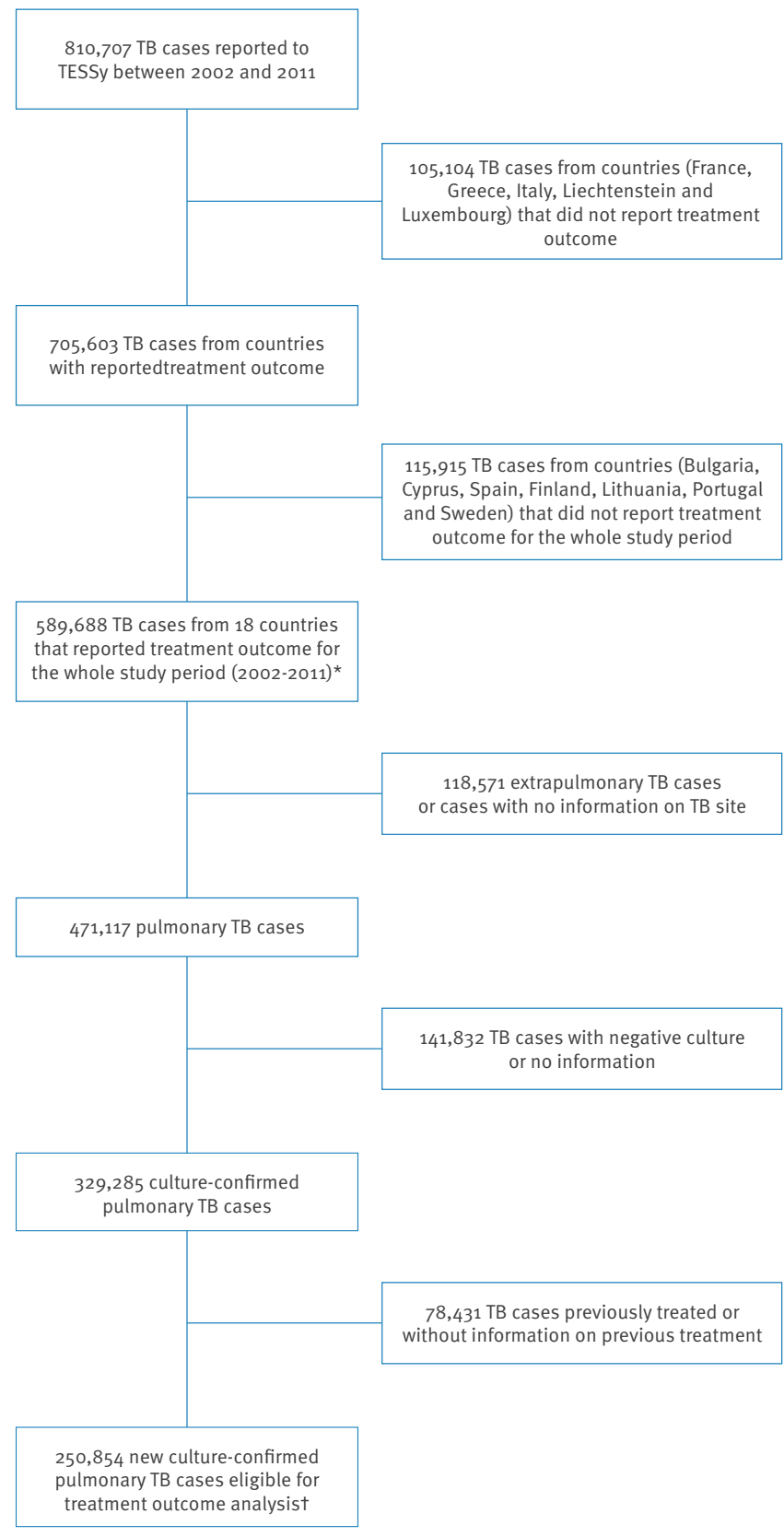

EEA: European Economic Area; EU: European Union

a Including Austria, Belgium, Czech Republic, Denmark, Estonia, Germany, Hungary, Ireland, Iceland, Latvia, Malta, the Netherlands, Norway, Poland, Romania, Slovenia, Slovakia and the United Kingdom.

b Denmark did not report information on previous treatment in 2005 . These cases were coded as missing in the cohort $(n=428)$.

Source: The European Surveillance System (TESSy). TESSy was operational since 2008 and for data from 2002-2007 data were transferred into TESSy from the historical database of the former EuroTB project for the TB surveillance activities in Europe.

\section{Methods}

\section{Data source}

Since January 2008, all EU countries (27; since February 201528 countries, after Croatia has joined) and the EEA countries Iceland and Norway (Liechtenstein has not reported data since 2007) report their available data on TB to The European Surveillance System (TESSy) hosted by ECDC [6]. For years between 1996 and 2007, data were transferred into TESSy from the historical database of the former EuroTB project for the TB surveillance activities in Europe. In this paper, we analysed data of TB TO during 2002-2011 and extracted from TESSy on 2 October 2013.

Since the reporting year 2002, case-based TO data have been provided by EU/EEA countries for cases notified one year before the year of TO reporting. Since 2005, TO data of MDR-TB cases have been reported for cases notified two years earlier [6]. Thus, for TO analysis of MDR-TB cases data reported between 2005 and 2010 were used in this study.

\section{Definitions}

In line with the WHO/IUATLD recommendation for EU/EEA countries $[3,4]$ the cohort eligible for the TO analysis included new culture-confirmed pulmonary TB cases. Cases are observed until the first outcome up to a maximum of 12 months after the start of treatment and MDR-TB cases for 24 months. TO was categorised based on the WHO/IUATLD recommendations [4] with two additional categories 'Still on treatment' and 'Unknown' (Table 1) [6,7].

A new TB case was defined as a case that never previously received drug treatment for active $\mathrm{TB}$, or received anti-TB drugs for less than one month. Five countries (Belgium, Denmark, Ireland, Norway and the United Kingdom (UK)) did not report information about previous treatment and for those previous diagnosis was used as a proxy for defining new and previously treated cases.

The term native case refers to cases born in or, if this information was unavailable, having citizenship in the reporting country. Foreign origin refers to cases born in (or citizens of) a country different from the reporting country.

MDR-TB was defined as TB with resistance to at least rifampicin and isoniazid. A low TB incidence country was defined as country with TB incidence rate $<20$ cases per 100,000 population and a high TB incidence country with TB incidence rate $\geq 20$ cases per 100,000 population.

\section{Statistical analysis}

Data were described by totals and percentages. The chi-squared test was used to assess differences in categorical variables. Only cases from countries 
Treatment outcome of new culture-confirmed pulmonary tuberculosis cases in the EU/EEA by A. Reporting years B. Reporting countries, 2002-2011 ( $\mathrm{n}=250,854)$

A.

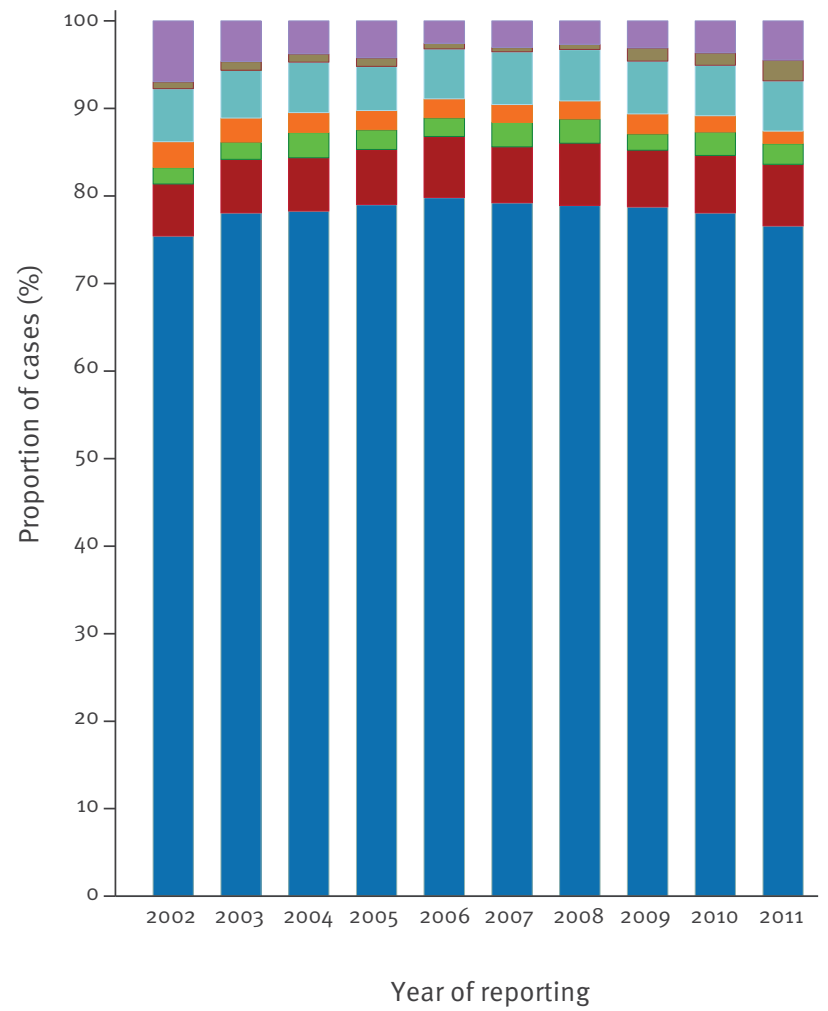

B.

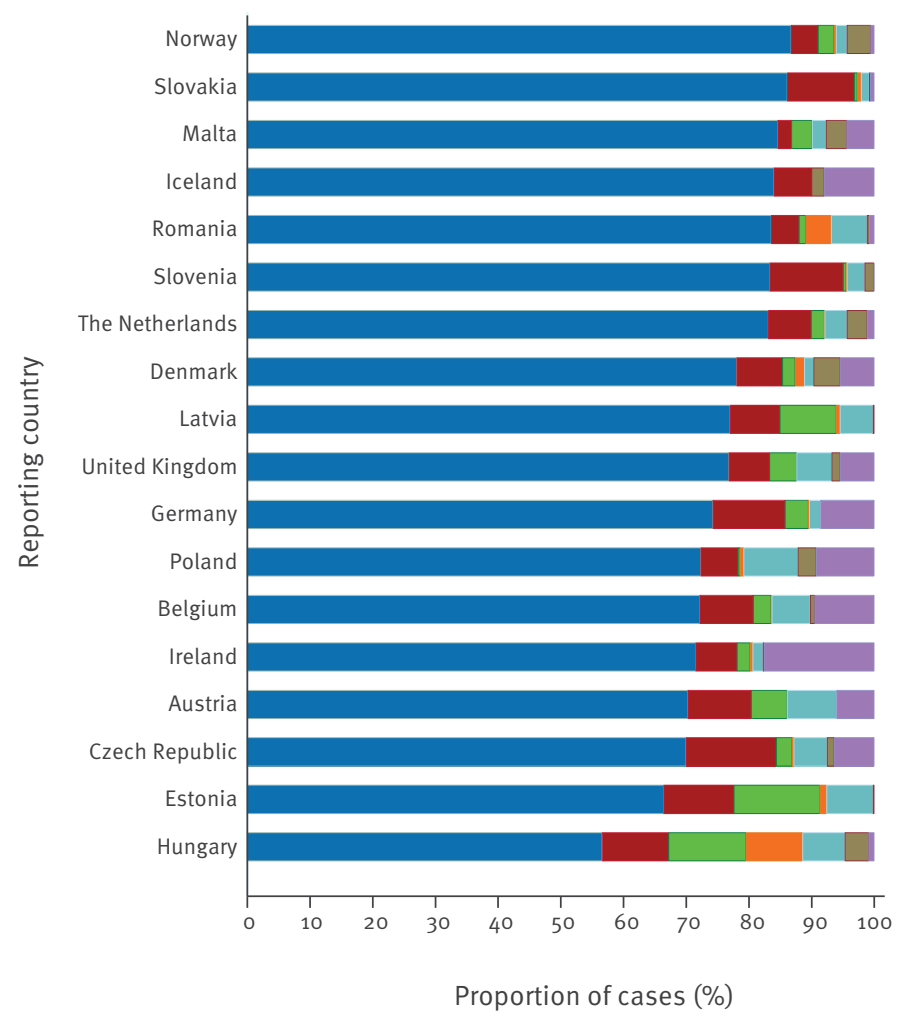

Still on treatment

- Transferred out Unknown outcome

Source: The European Surveillance System (TESSy). TESSy was operational since 2008 and for data from 2002-2007 data were transferred into TESSy from the historical database of the former EuroTB project for the TB surveillance activities in Europe.

that reported on TO for the whole study period were included in TO calculation and the statistical model.

Trend analysis of treatment success rate was conducted using the nonparametric test for trend across ordered groups i.e. the reporting years. A logistic regression model was used to examine the association between the TO, successful vs unsuccessful, and potential predictor variables age, sex, geographical origin, MDR-TB and reporting year. In the regression model, TB TO was categorised as treatment success vs unsuccessful treatment. Independent variables were systematically investigated in the univariate analysis and in the multivariable model for adjusting to possible confounders. To correct for the clustering within countries, we specified that the standard errors allow for intragroup correlation, assuming that the observations are independent across groups (clusters) but not necessarily within groups. Based on the regression coefficient of the model, odds ratios (OR) with their $95 \%$ confidence interval $(\mathrm{Cl})$ were also calculated to assess the strength of association.

All tests were two-sided and considered significant if $p<0.05$. All analyses were performed using STATA (version12, StataCorp, LP, TX, US) software.

\section{Ethical statement}

This study was based on national surveillance data submitted to ECDC. Therefore, written informed consent from the patients was not required due to the anonymous nature of the data.

\section{Results}

\section{Treatment outcome reporting}

Between 2002 and 2011, a total of 810,707 TB cases were notified. Of these, $83.3 \%(n=675,627)$ had information on TO. The number of countries, that reported information on TO, increased from 21 countries in 2002 to 25 countries in 2011. Five countries (France, Greece, 


\section{FIGURE 3}

Treatment success rate of new culture-confirmed pulmonary tuberculosis in the EU/EEA. 3A. Age group 3B. Treatment outcome by reporting year, 2002-2011 $(n=250,810)$

A.

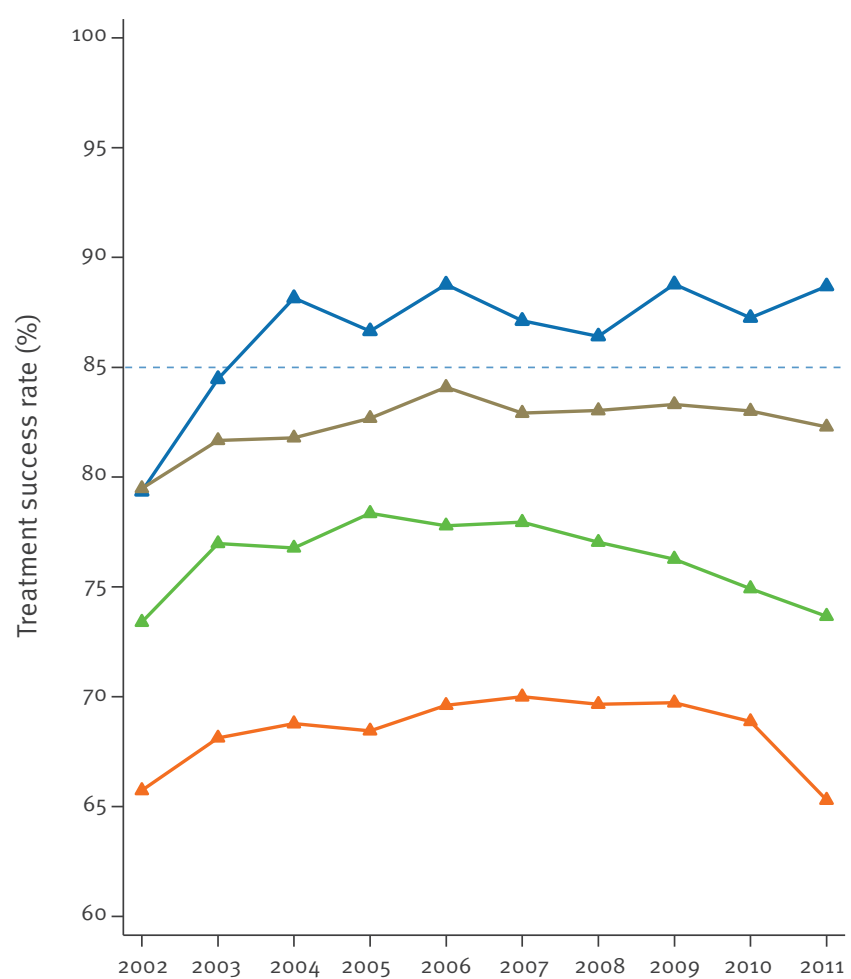

Year of reporting

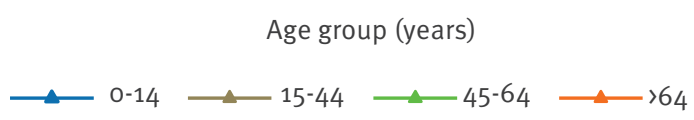

B.

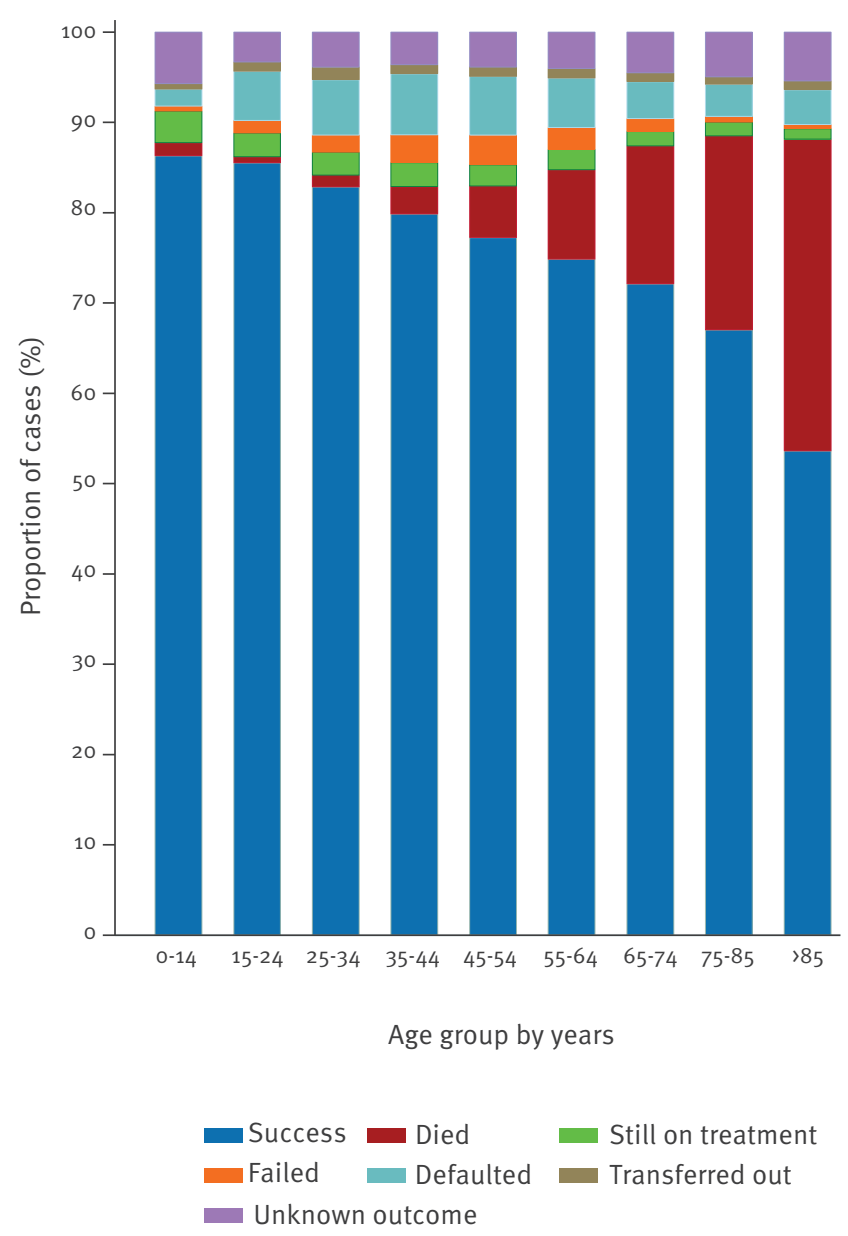

EU: European Union; EEA: European Economic Area

Source: The European Surveillance System (TESSy). TESSy was operational since 2008 and for data from 2002-2007 data were transferred into TESSy from the historical database of the former EuroTB project for the TB surveillance activities in Europe.

Italy, Liechtenstein and Luxembourg) did not report on TO in any year between 2002 and 2011. Eighteen countries (Austria, Belgium, Czech Republic, Denmark, Estonia, Germany, Hungary, Ireland, Iceland, Latvia, Malta, the Netherlands, Norway, Poland, Romania, Slovenia, Slovakia and the UK) provided information on TO for the whole 10-year study period (2002-2011), corresponding to 589,688 TB cases $(72.7 \%$ of all reported cases). Of these 589,688 TB cases, $42.5 \%$ $(n=250,854)$ were new culture-confirmed pulmonary TB cases eligible for our analysis (Figure 1).

Significant differences were observed in the demographic characteristics between included and excluded cases. Compared with the excluded cases, the included new culture-confirmed pulmonary TB cases were more likely to be men $(67.2 \%$ vs $63.7 \%$; p <0.001), of native origin ( $84.4 \%$ vs $78.7 \%$; $p<0.001$ ) and non-MDR-TB (98.6\% vs 97.9\%, p<0.001) respectively. Also, the treatment success rate was higher in included cases compared with excluded ones ( $78.2 \%$ vs $71.1 \%$; $p<0.001)$.

\section{Cohort characteristics}

The majority of cohort cases were reported by Romania, Poland, Germany and the UK (45.1\%, 17.8\%, 9.7\% and $9.5 \%$ respectively). The median age of cases was 45 years (interquartile range (IQR) 31-57). Of cohort cases $67.2 \%$ were male and $14.9 \%$ were of foreign origin. MDR-TB was reported in $1.4 \%(n=3,597)$ of the cohort cases, mainly from Romania $(n=1,219)$, Latvia $(n=802)$ and Estonia ( $\mathrm{n}=460)$. Information of drug susceptibility 


\section{FIGURE 4}

Treatment outcome at 24-month follow-up for new culture-confirmed pulmonary MDR-TB cases in the EU/ EEA, 2005-2010 $(n=2,140)$

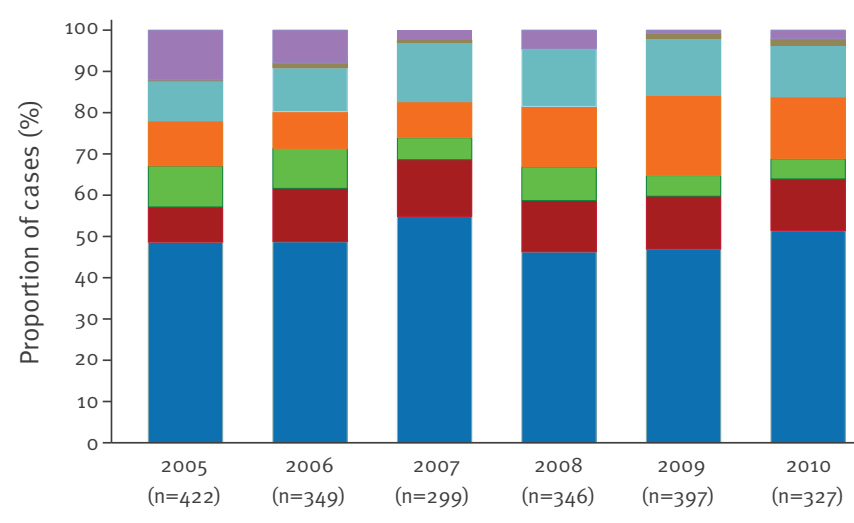

Reporting year

$$
\begin{aligned}
& \text { Success Died Still on treatment } \\
& \text { - Failed Defaulted Transferred out } \\
& \text { Unknown outcome }
\end{aligned}
$$

EU: European Union; EEA: European Economic Area; MDR: multidrug-resistant; TB: tuberculosis.

Source: The European Surveillance System (TESSy). TESSy was operational since 2008 and for data from 2002-2007 data were transferred into TESSy from the historical database of the former EuroTB project for the TB surveillance activities in Europe.

testing results for isoniazid and rifampicin was missing in almost half of the cases (48.4\%) (Table 2 ).

\section{Twelve-month treatment outcome}

The overall proportion of cases with reported treatment success was $78.2 \%$ (country range $56.6 \%$ in Hungary to $86.8 \%$ in Norway). Treatment success rate was higher among female cases compared with male cases $(81.6 \%$ vs $76.5 \%$ respectively; $p<0.01$ ) overall and in each respective reporting year, age group and geographical origin (data not shown). About $6.5 \%$ of TB cases died (country range $2.2 \%$ in Malta to $14.4 \%$ in Czech Republic). On average, $2.4 \%$ of cases were still on treatment at the end of the 12 -month follow-up period (country range $0 \%$ in Iceland to $13.7 \%$ in Estonia); $32.7 \%$ of these cases were MDR-TB. The proportion of cases with unsatisfactory outcome 12 months after start of treatment was $8.9 \%$ (country range $2.0 \%$ in Iceland and Germany to $19.6 \%$ in Hungary). Of them $2.2 \%$ were reported as treatment failure, $5.7 \%$ as defaulted and $1.0 \%$ as transferred out. Cases with unknown TO accounted for $4.0 \%$ (country range $0 \%$ in Latvia and Estonia to $17.6 \%$ in Ireland) (Figure 2).

The treatment success rate decreased with increasing age $(85.3 \%$ treatment success in cases aged $<15$ years vs $68.4 \%$ in cases aged $>64$ years; $p<0.001$ ) (Figure $3 a, b)$. The death rate rose substantially with increasing age of cases $(1.8 \%$ death rate in cases aged $<15$ years vs $19.4 \%$ in cases aged > 64 years; $p<0.001$ ) (Figure $3 b$ ).
A higher proportion of cases with unknown outcome was noticed among cases aged $<15$ years compared with other age groups.

The treatment success rate was slightly higher among native cases than among cases of foreign origin $(78.7 \%$ vs $77.3 \%$ respectively; $p=0.05)$. In native cases, a higher proportion of deaths $(6.9 \%$ in native cases vs $4.3 \%$ in cases of foreign origin; $p<0.001$ ) and treatment failure $2.7 \%$ in native cases vs $0.2 \%$ in cases of foreign origin; $p<0.001$ ) was observed. Also the proportion of cases with advanced age was higher in native cases than cases of foreign origin (cases aged $>64$ years accounted for $17.2 \%$ in native cases vs $10.8 \%$ in cases of foreign origin; $p<0.001$ ). The proportion of cases with unknown outcome was markedly higher in cases of foreign origin than in native cases (3.0 vs $6.5 \%$ respectively; $p<0.001$ ).

Adjusting for age and country of origin revealed no significant differences in treatment success rate between countries with low or high incidence (OR 0.7; $p=0.1$ ). Noticeably, the proportion of elderly aged $>64$ years was higher in low incidence countries compared with high incidence countries (cases aged $>64$ years accounted for $20.9 \%$ vs $14.6 \%$ respectively; $p<0.001$ ). Similarly, cases of foreign origin were more presented in low incidence countries $(44.7 \%$ vs $0.8 \%$ respectively; $p<0.001$ )

\section{Twenty-four-month treatment outcome of multidrug-resistant tuberculosis}

Between 2005 and 2010, 2,140 MDR-TB cases were reported from the $18 \mathrm{EU} / \mathrm{EEA}$ countries included in the analysed cohort. The overall treatment success rate for MDR-TB cases at 24-month follow-up was $49.2 \%$ and did not reach the $70 \%$ target of treatment success rate for MDR-TB cases in any year of the observation period. About $12.0 \%$ of MDR-TB cases died and $7.3 \%$ were still on treatment at the end of 24-month follow-up. The proportion of cases with unsatisfactory outcome was $26.3 \%$ (including $13.1 \%$ failed, $12.4 \%$ defaulted and $0.8 \%$ transferred out). About $5.2 \%$ of the MDR-TB cases were reported with unknown TO (Figure 4).

\section{Trend analysis}

The $85 \%$ target of overall treatment success rate was not reached in any year between 2002 and 2011. Analysis of trends shows that the treatment success rate increased from $75.4 \%$ in 2002 to $79.8 \%$ in 2006 $(p=0.04)$, then subsequently decreased to $76.6 \%$ in $2011(p=0.07)$. No change in the cohort profile regarding proportions of age groups, sex and MDR-TB was observed over time. The proportion of cases of foreign origin increased from $(\mathrm{N}=3672 ; 14.5 \%)$ in 2006 to $(\mathrm{N}=4081 ; 18.5 \%)$ in 2011 ( $\mathrm{p}<0.001)$. Some countries (Belgium, Germany, Hungary, Romania and the UK) showed a continuous increase in the treatment success rate throughout the observation period, while for other countries (Austria, Denmark, Estonia, Netherlands and Poland) a decline was observed (data not shown). 
TABLE 1

Summary of tuberculosis treatment outcome categories according to the WHO/IUATLD recommendations and the ECDC surveillance report 2015

\begin{tabular}{|l|l|}
\hline Categories & Definitions \\
\hline Cured & $\begin{array}{l}\text { Treatment completion and culture-negative samples taken at the end of treatment and on at } \\
\text { least one previous occasion. }\end{array}$ \\
\hline Completed & $\begin{array}{l}\text { Treatment completed, but case does not meet the criteria to be classified as cured or } \\
\text { treatment failure. }\end{array}$ \\
\hline Failed & $\begin{array}{l}\text { Culture or sputum smear remaining positive or becoming positive again five months or later } \\
\text { into the course of treatment. }\end{array}$ \\
\hline Died & Death before cured or treatment completion, irrespective of cause. \\
\hline Defaulted & $\begin{array}{l}\text { Treatment interrupted for two months or more, not resulting from a decision of the care } \\
\text { provider. }\end{array}$ \\
\hline Transferred out & $\begin{array}{l}\text { Patient referred to another clinical unit for treatment and information on outcome not } \\
\text { available. }\end{array}$ \\
\hline Still on treatment & $\begin{array}{l}\text { Patient still on treatment at 12 months and at 24 months for MDR-TB cases without any } \\
\text { other outcome during treatment. }\end{array}$ \\
\hline Unknown outcome & Information on outcome not available, for cases not known to have been transferred. \\
\hline Groups created for the purpose of this study & \multicolumn{2}{|l|}{} \\
\hline Unsatisfactory outcome & $\begin{array}{l}\text { The percentage of cases that interrupted treatment, were transferred or failed treatment } \\
\text { out of all notified. }\end{array}$ \\
\hline Successful outcome/treatment success & The percentage of cases that were cured or completed treatment out of all notified. \\
\hline Unsuccessful treatment & $\begin{array}{l}\text { The percentage of cases that were not cured or completed treatment out of all notified (i.e. } \\
\text { unsatisfactory outcome, death, still on treatment and unknown outcome). }\end{array}$ \\
\hline
\end{tabular}

ECDC: European Centre for Disease Prevention and Control; IUATLD: International Union against Tuberculosis and Lung Disease; WHO: World Health Organization.

Factors associated with unsuccessful treatment In the multivariable logistic regression model, unsuccessful treatment was significantly associated with increasing age of the case (adjusted OR $(\mathrm{aOR})=1.02$ per a one-year increase, $95 \% \mathrm{Cl}$ : $1.02-1.02)$, male sex $(\mathrm{aOR}=1.40,95 \% \mathrm{Cl}: 1.28-1.52)$ and foreign origin $(\mathrm{aOR}=1.32,95 \% \mathrm{Cl}: 1.03-1.70)$. The strongest association with unsuccessful treatment was observed with MDR-TB (aOR=8.7, 95\%Cl: 5.09-14.97). No association was found between unsuccessful treatment and reporting period $(\mathrm{aOR}=0.96,95 \% \mathrm{Cl}: 0.71-1.30)$ (Table 3). In a separate analysis using a multivariable multinomial model, we did not find any difference in factors associated with death and those associated with the other unsuccessful treatment outcomes i.e. unsatisfactory outcome, still on treatment and unknown outcome (data not shown).

\section{Discussion}

This study investigated TB TO and factors associated with unsuccessful treatment in the EU/EEA over 10 years following the WHO/IUATLD recommendations for cohort analysis. It shows that the overall treatment success rate of new culture-confirmed pulmonary TB cases in the EU/EEA was $78.2 \%$ and failed to reach the $85 \%$ target in any year between 2002 and 2011. The study also indicates that main factors associated with unsuccessful treatment were increasing age, male sex, foreign origin and MDR-TB.
Still some countries do not provide information on TO to TESSy and for those who reported on TO, the proportion of cases with unknown outcome remained high at $4.0 \%$ overall. It is true that an unknown TO does not necessarily represent a negative one, yet from a programmatic perspective, lack of knowledge about TO deprives the programme from important information to guide TB control. Moreover, no significant improvement in treatment success rate was observed over the 10-year study period. These findings demonstrate a programmatic weakness within TB control in the EU/ EEA and highlight the urgent need for strengthening the monitoring and evaluation process at country level [8].

Another analysis of data in TESSy reported by $22 \mathrm{EU} /$ EEA countries in 2007 , showed a treatment success rate of $79.5 \%$ for new culture-confirmed pulmonary cases [8]. A meta-analysis of published reports of TB TO in Europe found that $74.4 \%$ of outcomes were successful [9]. Another study conducted in 18 EU/EEA countries in 2005 , showed a mean success rate of only $69 \%$ [10]. These results need to be compared with caution due to the different definition of cohort used and the different study periods [11]. Higher treatment success rates were reported in low incidence countries in North America, with $89 \%$ in the United States (US) in 2013, and with $86 \%$ in 2012 in Canada [12,13].

In the multivariable regression model, male sex was independently associated with unsuccessful treatment. The lower treatment success rate in men compared 
TABLE 2 A* $^{*}$

Characteristics of new pulmonary culture-positive tuberculosis cases in the EU/EEA ${ }^{\mathrm{a}}, 2002-2011(\mathrm{n}=250,854)$

\begin{tabular}{|c|c|c|}
\hline $\begin{array}{l}\text { Demographic and clinical } \\
\text { features of TB cases }\end{array}$ & $\begin{array}{l}\text { Number of cases } \\
(n=250,854 \text { cases })\end{array}$ & Percent \\
\hline \multicolumn{3}{|l|}{ Sex } \\
\hline Female & 82,336 & 32.8 \\
\hline Male & 168,442 & 67.2 \\
\hline Unknown & 76 & 0.03 \\
\hline \multicolumn{3}{|l|}{ Age group } \\
\hline$\ll 15$ & 3,477 & 1.4 \\
\hline $15-44$ & 125,800 & 50.2 \\
\hline $45-64$ & 79,815 & 31.8 \\
\hline$>64$ & 41,718 & 16.6 \\
\hline Unknown & 44 & 0.02 \\
\hline \multicolumn{3}{|l|}{ Geographical origin } \\
\hline Native cases & 202,129 & 80.6 \\
\hline Cases of foreign origin & 37,471 & 14.9 \\
\hline Unknown & 11,254 & 4.5 \\
\hline \multicolumn{3}{|l|}{ HIV status } \\
\hline Positive & 623 & 0.3 \\
\hline Negative & 14,889 & 5.9 \\
\hline Unknown & 235,342 & 93.8 \\
\hline \multicolumn{3}{|l|}{ Multidrug-resistance } \\
\hline No & 125,880 & 50.2 \\
\hline Yes & 3,597 & 1.4 \\
\hline Unknown & 121,377 & 48.4 \\
\hline \multicolumn{3}{|l|}{ EU/EEA countries } \\
\hline $\begin{array}{l}\text { Countries with low TB incidence } \\
\text { rate }^{b}\end{array}$ & 82,711 & 33.0 \\
\hline $\begin{array}{l}\text { Countries with high TB incidence } \\
\text { rate }^{c}\end{array}$ & 168,143 & 67.0 \\
\hline
\end{tabular}

Countries reporting treatment outcome for the whole study period

\begin{tabular}{|l|c|c|}
\hline Austria & 4,030 & 1.6 \\
\hline Belgium & 4,922 & 2.0 \\
\hline Czech Republic & 4,911 & 2.0 \\
\hline Denmark & 1,928 & 0.8 \\
\hline Estonia & 2,771 & 1.1 \\
\hline Germany & 24,195 & 9.7 \\
\hline Hungary & 6,347 & 2.5 \\
\hline Ireland & 1,704 & 0.7 \\
\hline Iceland & 50 & 0.02 \\
\hline Latvia & 7,727 & 3.1 \\
\hline Malta & 91 & 0.1 \\
\hline The Netherlands & 5,205 & 2.1 \\
\hline
\end{tabular}

EU: European Union; EEA: European Economic Area; TB: tuberculosis; UK: United Kingdom.

a Including 18 countries that reported treatment outcome for the whole study period: Austria, Belgium, Czech Republic, Denmark, Estonia, Germany, Hungary, Ireland, Iceland, Latvia, Malta, the Netherlands, Norway, Poland, Romania, Slovenia, Slovakia and the United Kingdom.

${ }^{b}$ Countries with low TB incidence rate $(<20,000 / 100,000$ population) included Austria, Belgium, Czech Republic, Denmark, Germany, Hungary, Ireland, Iceland, Malta, the Netherlands, Norway, Slovenia, Slovakia and UK.

c Countries with high TB incidence rate $(\geq 20,000 / 100,000$ population) included Estonia, Latvia, Poland and Romania.

Source: The European Surveillance System (TESSy). TESSy was operational since 2008 and for data from 2002-2007 data were transferred into TESSy from the historical database of the former EuroTB project for the TB surveillance activities in Europe.
TABLE 2 B

Characteristics of new pulmonary culture-positive tuberculosis cases in the EU/EEA ${ }^{\mathrm{a}}, 2002-2011(\mathrm{n}=250,854)$

\begin{tabular}{|l|c|c|}
\hline $\begin{array}{l}\text { Demographic and clinical } \\
\text { features of TB cases }\end{array}$ & \multicolumn{1}{c|}{$\begin{array}{c}\text { Number of cases } \\
(n=250,854 \text { cases })\end{array}$} & Percent \\
\hline Countries reporting treatment outcome for the whole study period \\
\hline Norway & 1,216 & 0.5 \\
\hline Poland & 44,577 & 17.8 \\
\hline Romania & 113,068 & 45.1 \\
\hline Slovenia & 1,679 & 0.7 \\
\hline Slovakia & 2.553 & 1.0 \\
\hline UK & 23,880 & 9.5 \\
\hline Treatment outcome & 196,105 & 78.2 \\
\hline $\begin{array}{l}\text { Success (cured or treatment } \\
\text { completed) }\end{array}$ & 16,222 & 6.5 \\
\hline Died & 5,914 & 2.4 \\
\hline $\begin{array}{l}\text { Still on treatment (12 months } \\
\text { follow-up) }\end{array}$ & 5,583 & 2.2 \\
\hline Failed & 14,392 & 5.7 \\
\hline Defaulted & 2,653 & 1.0 \\
\hline Transferred out & 9,985 & 4.0 \\
\hline Unknown outcome & & \\
\hline
\end{tabular}

EU: European Union; EEA: European Economic Area; TB: tuberculosis; UK: United Kingdom.

a Including 18 countries that reported treatment outcome for the whole study period: Austria, Belgium, Czech Republic, Denmark, Estonia, Germany, Hungary, Ireland, Iceland, Latvia, Malta, the Netherlands, Norway, Poland, Romania, Slovenia, Slovakia and the United Kingdom.

${ }^{b}$ Countries with low TB incidence rate $(<20,000 / 100,000$ population) included Austria, Belgium, Czech Republic, Denmark, Germany, Hungary, Ireland, Iceland, Malta, the Netherlands, Norway, Slovenia, Slovakia and UK.

c Countries with high TB incidence rate ( $\geq 20,000 / 100,000$ population) included Estonia, Latvia, Poland and Romania.

Source: The European Surveillance System (TESSy). TESSy was operational since 2008 and for data from 2002-2007 data were transferred into TESSy from the historical database of the former EuroTB project for the TB surveillance activities in Europe.

with women was mostly attributed to a higher proportion of died, failed and defaulted in men compared with women, which persisted when stratifying by reporting year, country of origin and age group (with exception among cases aged $<15$ years). Another study done in the EU reported also that the success rate was higher in women and this was due to a greater occurrence of defaulted and treatment failure in men [10]. A study from South Africa found that men were less likely to adhere to their treatment than women and male sex was associated with the risk of treatment interruption [14]. The poorer TO in men can be attributed to some behavioural components such as alcohol and drug abuse, which are still predominant among men. Studies done in Paris [15] and Hamburg [16] indicated that unsuccessful TB treatment was associated with alcohol and injecting drug use. A Spanish study showed that injecting drug use was associated with treatment default; while alcoholism was associated with death during TB treatment [17]. According to the European 
Factors associated with treatment outcome among new culture-confirmed pulmonary tuberculosis cases, 2002-2011 $(\mathrm{n}=250,854)$

\begin{tabular}{|c|c|c|c|c|c|c|}
\hline \multirow{2}{*}{ Predicting factors } & \multicolumn{2}{|c|}{$\begin{array}{l}\text { Treatment success } \\
\text { (totals) }\end{array}$} & \multicolumn{2}{|c|}{ Univariable analysis ${ }^{a}$} & \multicolumn{2}{|c|}{ Multivariable analysisa,b } \\
\hline & Yes & No & $\begin{array}{c}\text { OR } \\
(95 \% \mathrm{Cl})\end{array}$ & $p$ value & $\begin{array}{c}\text { AOR } \\
(95 \% \mathrm{Cl})\end{array}$ & $\mathrm{p}$ value \\
\hline $\begin{array}{l}\text { Increase in age per year } \\
\text { (continues variable) }\end{array}$ & 196,895 & 53,959 & $1.02(1.01-1.02)$ & $<0.001$ & $1.02(1.02-1.02)$ & $<0.001$ \\
\hline \multicolumn{7}{|l|}{ Sex } \\
\hline Female & 67,512 & 14,824 & 1 & \multirow{2}{*}{$<0.001$} & 1 & \multirow{2}{*}{$<0.001$} \\
\hline Male & 129,323 & 39,119 & $1.38(1.27-1.49)$ & & $1.40(1.28-1.52)$ & \\
\hline \multicolumn{7}{|l|}{ Country of origin } \\
\hline Native cases & 159,617 & 42,512 & 1 & \multirow{2}{*}{0.72} & 1 & \multirow{2}{*}{0.03} \\
\hline Cases of foreign origin & 29,151 & 8,320 & $1.07(0.74-1.56)$ & & $1.32(1.03-1.70)$ & \\
\hline \multicolumn{7}{|l|}{ Multidrug-resistance } \\
\hline No & 99,666 & 26,214 & 1 & \multirow{2}{*}{$<0.001$} & 1 & \multirow{2}{*}{$<0.001$} \\
\hline Yes & 1,252 & 2,345 & $7.12(4.07-12.44)$ & & $8.7(5.09-14.97)$ & \\
\hline Unknown & 95,977 & 25,400 & $1.00(0.77-1.32)$ & 0.96 & $1.08(0.86-1.36)$ & 0.51 \\
\hline \multicolumn{7}{|l|}{ Reporting year } \\
\hline 2002 to 2006 & 105,566 & 29,445 & 1 & \multirow{2}{*}{0.73} & 1 & \multirow{2}{*}{0.81} \\
\hline 2007 to 2011 & 91,329 & 24,514 & $0.96(0.77-1.19)$ & & $0.96(0.71-1.30)$ & \\
\hline
\end{tabular}

EU: European Union; EEA: European Economic Area; MDR: multidrug-resistant; TB: tuberculosis.

annivariable and multivariable analysis were performed using logistic regression models specifying that standard errors allow for intracountry correlation (controlling for clustering within countries).

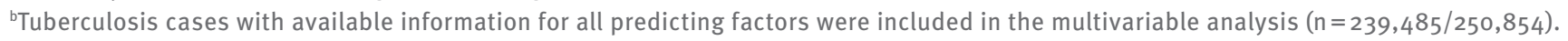

The logistic regression models were based on 12-month outcomes for non-MDR TB cases and on 24-month outcomes for MDR-TB cases.

Source: The European Surveillance System (TESSy). TESSy was operational since 2008 and for data from 2002-2007 data were transferred into TESSy from the historical database of the former EuroTB project for the TB surveillance activities in Europe.

Monitoring Centre for Drugs and Drug Addiction, drug use and alcohol consumption are considerably more common among men than women in EU countries [18]. Biological factors may also contribute to the different TB TO between males and females. Animal models showed that male mice developed more severe TB disease, while females were more resistant and exhibit more robust immune responses to infection [19].

In our study, the proportion of cases with unsatisfactory outcomes was $8.9 \%$; thus below the maximum proportion of $10 \%$ in the WHO/IUATLD recommendations. This threshold aims to serve National TB Programmes as an orientation to direct their efforts in improving TO [4]. Three countries: Hungary, Poland and Romania, exceeded the $10 \%$ proportion of unsatisfactory outcomes. The proportion of deaths (any cause) was on average $6.5 \%$. A high proportion of deaths among pulmonary TB cases $(10 \%)$ was also reported by Canada in 2012 [13].

An inverse relationship between treatment success rate and increasing age was observed in this study. This can be partially explained by the increasing death rate with advancing age; as a result of demographic changes with an ageing population. However, studies from high- and low-incidence countries reported that older age also increases the risk of unsuccessful TB treatment [20-22]. The elderly are at increased risk for missed diagnosis [23] due to the fact that a diagnosis of TB among the elderly is often difficult due to atypical, non-specific clinical manifestation and may be confused with other concomitant age-related diseases $[24,25]$. This can lead to a delayed diagnosis and more advanced disease at presentation which in turn leads to increased mortality among the elderly $[24,25]$. A study in Norway demonstrated that deaths occurred mainly because TB diagnosis was established too late and half of the cases were only detected at autopsy [26]. Also a study from the US found that advanced age was strongly associated with unrecognised pulmonary TB leading to premature death [27]. Therefore, a high index of suspicion for TB in the elderly is undoubtedly justifiable [23]. Most recently, the WHO Framework towards TB elimination in low-incidence countries highlighted the particular challenges in treating active TB in the elderly and the importance of early diagnoses among them [28]. Furthermore, screening for active TB among the elderly has been suggested by WHO depending on the local TB epidemiology and risk-benefit assessments [28]. At present in Japan, early case detection and treatment are considered as the most effective TB control measures among the elderly in order to protect them from TB death [29].

In our analysis, MDR-TB was the strongest risk factor for unsuccessful treatment and treatment success rate of MDR-TB cases at 24-month follow-up was $49.2 \%$, far 
below the $70 \%$ target, a sign of the seriousness of the MDR-TB epidemic in the EU/EEA. It is well documented that treatment failure and mortality are higher among MDR-TB cases than among susceptible cases [30,31].

Although we found only a slight difference in treatment success rate in native and foreign cases, being of foreign origin was a significant risk factor for unsuccessful TO in our analysis. Markedly, a high proportion of cases of foreign origin had unknown outcome. It is unclear to which extent this might be due to migration for medical reasons. Overall, this result suggests that programmatic issues may play a role including access to healthcare in the context of mobility e.g. patients returning to their country of origin before the treatment is completed, as was observed in a study from London [32], and challenges in cross-border collaboration. Thus, the 61st World Health Assembly in 2008 called on countries to address migrant health issues in a more integrated, harmonised approach [33]. Foreign origin may be a proxy for other unmeasured indicators related to migration. In the EU, migrants have been reported to be at risk of not receiving the same level of healthcare in the preventive, diagnostic and treatment services as the native communities [34]. This might be due to a combination of factors including legal and working status, social exclusion, substandard economic condition and barriers in accessing healthcare services [34].

\section{Limitations}

This study has a number of limitations. A considerable proportion of reported cases was without information on TO and was therefore excluded from the analysis. Following the WHO/IUATLD recommendation for TO monitoring in the EU/EEA, only new cultureconfirmed pulmonary TB cases were eligible for the analysis cohort, accounting for $42 \%$ of reported cases. Furthermore, a higher treatment success rate was found among included cases compared to excluded ones. Our results thus reflect the situation of the defined cohort and might overestimate the overall treatment success rate in the EU/EEA. A possible selection bias related to reporting countries can be noticed; some countries were overrepresented in our cohort e.g. Romania accounted for $45 \%$ of the cases included. Information on drug susceptibility testing results was missing for almost half of cases, some of whom may have been MDR-TB. Due to the unavailability of cause of death information in the database, we could not distinguish between cases who died from TB or due to other causes during TB treatment. This would be of particular importance for analysing TO among the elderly. With the data currently reported to TESSy, it is not possible to investigate some factors known to be associated with TB TO such as comorbidity (like HIV infection), drug or alcohol abuse, homelessness and other socioeconomic factors. These underlying factors are important for mapping risk groups in order to improve TB prevention and access to TB services, since TB is concentrated in certain at-risk groups in most lowincidence countries [28]. Poor implementation of TB programme guidelines in some EU/EEA countries might partially account for suboptimal TO, especially among MDR-TB cases. However, the degree of implementation of guidelines within countries and the appropriateness of the guidelines are beyond the scope of this paper.

\section{Conclusion}

In conclusion, shortcomings in TO reporting remain a challenge in monitoring TB control in the EU/EEA. The treatment success rate was $78 \%$, below the global target of $85 \%$ for the decade after 2002. Special efforts are required for patients with MDR-TB, who have particularly low treatment success rates. For elderly patients, a high index of clinical suspicion for TB is required to ensure early diagnosis and treatment of TB [24], and improve TO among them. Finally, to allow for valid monitoring at EU/EEA level, all countries should aim to report TO for all TB cases. Collecting additional variables on social determinants, alcohol and drug abuse, and co-morbidities could increase our understanding of the factors related to TB TO and support the design of appropriate interventions.

\section{${ }^{\star}$ Erratum}

Table 2 had to be replaced due to technical issues on 11 December 2015.

\section{Acknowledgements}

The authors would like to acknowledge the work of the ECDC national surveillance focal points and the TB national surveillance network who make EU/EEA TB surveillance possible. The authors acknowledge the nominated national operational contact points for tuberculosis Peter Henrik Andersen, Delphine Antoine, Trude Margrete Arnesen, Thorsteinn Blondal, Domnica Ioana Chiotan, Edita Davidavičienè, Irene Demuth, Raquel Duarte, Sabine Erne, Walter Haas, Alexander Indra, Jerker Jonsson, Ourania Kalkouni, Maria Koliou, Maria Korzeniewska - Kosela, Gábor Kovács, Joan O’Donnell, Analita Pace Asciak, Maria Grazia Pompa, Elena Rodríguez Valín, Erika Slump, Hanna Soini, Ivan Solovič, Petra Svetina, Lucy Thomas, Tonka Varleva, Piret Viiklepp, Kate Vulāne, Jiř̌i Wallenfels and Maryse Wanlin for providing the surveillance data used in this analysis.

Source of funding: The German Federal Ministry of Health is acknowledged for funding this study (grant number: IIA52513NIKo01//321-4471-02/). The funders had no role in study design, data collection and analysis, decision to publish, or preparation of the manuscript.

\section{Conflict of interest}

The authors declare that they have no competing interests.

\section{Authors' contributions}

Concept and design (BK, WH, BH, MvdW), statistical analysis (BK), interpretation of the data $(\mathrm{BK}, \mathrm{VH})$, drafting the manuscript (BK) and critical revision of the manuscript for important intellectual content (BK, WH, BH, MvdW, VH, LF). All authors read and approve the final manuscript. 


\section{References}

1. World Health Organization (WHO). 44th World Health Assembly, Resolutions and Decisions. Resolution WHA44.8. Geneva: WHO; 1991. Available from: http://www.who.int/tb/ publications/tbresolution_wha44_8_1991.pdf?ua=1

2. Stop TB Partnership and World Health Organization. Global Plan to Stop TB 2006-2015: Actions for Life - Towards a world free of tuberculosis. WHO/HTM/STB/2006.35. Geneva: WHO; 2006. http://www.stoptb.org/assets/documents/global/plan/ globalplanfinal.pdf

3. Rieder HL, Watson JM, Raviglione MC, Forssbohm M, Migliori GB, Schwoebel V, et al. Surveillance of tuberculosis in Europe. Working Group of the World Health Organization (WHO) and the European Region of the International Union Against Tuberculosis and Lung Disease (IUATLD) for uniform reporting on tuberculosis cases. Eur Respir J. 1996;9(5):1097-104. Available from: DOI: 10.1183/09031936.96.09051097 PMID: 8793477

4. Veen J, Raviglione M, Rieder HL, Migliori GB, Graf P, Grzemska $M$, et al. Standardized tuberculosis treatment outcome monitoring in Europe. Recommendations of a Working Group of the World Health Organization (WHO) and the European Region of the International Union Against Tuberculosis and Lung Disease (IUATLD) for uniform reporting by cohor analysis of treatment outcome in tuberculosis patients. Eur Respir J. 1998;12(2):505-10. Available from: DOI: 10.1183/09031936.98.12020505 PMID: 9727811

5. European Centre for Disease Prevention and Control (ECDC). A Framework Action Plan to fight Tuberculosis in the EU. Stockholm: ECDC; 2008. Available from: http://ecdc.europa. eu/en/publications/Publications/0803_SPR_TB_Action_plan. pdf

6. European Centre for Disease Prevention and Control/WHO Regional Office for Europe (ECDC/WHO EURO). Tuberculosis surveillance and monitoring in Europe 2015. Stockholm: ECDC; 2015. Available from: http://ecdc.europa.eu/en/publications/ Publications/tuberculosis-surveillance-monitoringEurope-2015.pdf

7. Falzon D, Scholten J, Infuso A. Tuberculosis outcome monitoring--is it time to update European recommendations?Euro Surveill. 2006;11(3):20-5.PMID: 16567878

8. Manissero D, Hollo V, Huitric E, Kodmon C, Amato-Gauci A. Analysis of tuberculosis treatment outcomes in the European Union and European Economic Area: efforts needed towards optimal case management and control.Euro Surveill. 2010;15(11).PMID: 20338143

9. Faustini A, Hall AJ, Perucci CA. Tuberculosis treatment outcomes in Europe: a systematic review. Eur Respir J. 2005;26(3):503-10. Available from: DOI: 10.1183/09031936.05.00103504 PMID: 16135735

10. Falzon D, Le Strat Y, Belghiti F, Infuso A, Euro TB. Correspondents. Exploring the determinants of treatment success for tuberculosis cases in Europe.Int J Tuberc Lung Dis. 2005;9(11):1224-9.PMID: 16333929

11. van Hest R, Ködmön C, Verver S, Erkens CG, Straetemans $M$, Manissero D, et al. Tuberculosis treatment outcome monitoring in European Union countries: systematic review. Eur Respir J. 2013;41(3):635-43. Available from: DOI: 10.1183/09031936.00030612 PMID: 22790913

12. Centers for Disease Control and Prevention (CDC). Reported Tuberculosis in the United States, 2013. Atlanta: CDC; October 2014. Available from: http://www.cdc.gov/tb/statistics/ reports/2013/pdf/report2013.pdf

13. Public Health Agency of Canada. Tuberculosis in Canada 2012 - Pre-Release. Ottawa (Canada): Minister of Public Works and Government Services Canada; 2014. Available from: http:// www.phac-aspc.gc.ca/tbpc-latb/pubs/tbcan13pre/assets/pdf/ tbcan13pre-eng.pdf

14. Connolly C, Davies GR, Wilkinson D. Who fails to complete tuberculosis treatment? Temporal trends and risk factors for treatment interruption in a community-based directly observed therapy programme in a rural district of South Africa.Int Tuberc Lung Dis. 1999;3(12):1081-7.PMID: 10599011

15. Valin N, Hejblum G, Borget I, Mallet HP, Antoun F, Che D, et al. Management and treatment outcomes of tuberculous patients, eastern Paris, France, 2004. Int J Tuberc Lung Dis. 2009;13(7):881-7.PMID: 19555539

16. Diel R, Niemann S. Outcome of tuberculosis treatment in Hamburg: a survey, 1997-2001.Int J Tuberc Lung Dis. 2003;7(2):124-31.PMID: 12588012

17. Working Group on Completion of Tuberculosis Treatment in Spain,Caylà JA, Caminero JA, Rey R, Lara N, Vallés X, GaldósTangüis H. Current status of treatment completion and fatality among tuberculosis patients in Spain.Int J Tuberc Lung Dis. 2004;8(4):458-64.PMID: 15141739
18. European Monitoring Centre for Drugs and Drug Addiction (EMCDDA). Selected Issue 2: A gender perspective on drug use and responding to drug problems. ISBN 92-9168-250-0. Lisbon: EMCDDA; 2006. Available from: http://www.drugs. ie/resourcesfiles/ResearchDocs/Europe/Research/2006/ sel2006_2-en.pdf

19. Nhamoyebonde S, Leslie A. Biological differences between the sexes and susceptibility to tuberculosis.J Infect Dis. 2014;209(3) Suppl 3;S100-6. Available from: DOI: 10.1093/ infdis/jiu147 PMID: 24966189

20. Lefebvre N, Falzon D. Risk factors for death among tuberculosis cases: analysis of European surveillance data. Eur Respir J. 2008;31(6):1256-60. Available from: DOI: 10.1183/09031936.00131107 PMID: 18515556

21. Bustamante-Montes LP, Escobar-Mesa A, Borja-Aburto VH, Gómez-Muñoz A, Becerra-Posada F. Predictors of death from pulmonary tuberculosis: the case of Veracruz, Mexico.Int J Tuberc Lung Dis. 2000;4(3):208-15.PMID: 10751065

22. Hauer B, Brodhun B, Altmann D, Fiebig L, Loddenkemper $R$, Haas W. Tuberculosis in the elderly in Germany. Eur Respir J. 2011;38(2):467-70. Available from: DOI: 10.1183/09031936.00199910 PMID: 21804163

23. Rajagopalan S. Tuberculosis and aging: a global health problem.Clin Infect Dis. 2001;33(7):1034-9. Available from: DOI: $10.1086 / 322671$ PMID: 11528577

24. Packham S. Tuberculosis in the elderly.Gerontology. 2001;47(4):175-9. Available from: DOI: 10.1159/000052794 PMID: 11408720

25. Zevallos M, Justman JE. Tuberculosis in the elderly.Clin Geriatr Med. 2003;19(1):121-38. Available from: DOI: 10.1016/So7490690(02)00057-5 PMID: 12735118

26. Naalsund A, Heldal E, Johansen B, Kongerud J, Boe J. Deaths from pulmonary tuberculosis in a low-incidence country.J Intern Med. 1994;236(2):137-42. Available from: DOI: 10.1111/ j.1365-2796.1994.tb01275.x PMID: 8046312

27. Rieder HL, Kelly GD, Bloch AB, Cauthen GM, Snider DE. Tuberculosis diagnosed at death in the United States. Chest. 1991;100(3):678-81. Available from: DOI: 10.1378 chest.100.3.678 PMID: 1889256

28. World Health Organization (WHO). Framework towards tuberculosis elimination in low-incidence countries. WHO HTM/TB/2014.13. Geneva: WHO; 2014. Available from: http:// apps.who.int/iris/bitstream/10665/132231/1/9789241507707_ eng.pdf?ua=1

29. Ohmori M, Wada M, Yoshiyama T, Uchimura K. (Factors related to early case detection of tuberculosis in health service facilities for the elderly).Kekkaku. 2003;78(6):435-42.PMID: 12872702

30. Espinal MA, Kim SJ, Suarez PG, Kam KM, Khomenko AG, Migliori GB, et al. Standard short-course chemotherapy for drug-resistant tuberculosis: treatment outcomes in 6 countries. JAMA. 2000;283(19):2537-45. Available from: DOI: 10.1001/ jama.283.19.2537 PMID: 10815117

31. Noeske J, Nguenko PN. Impact of resistance to antituberculosis drugs on treatment outcome using World Health Organization standard regimens.Trans R Soc Trop Med Hyg. 2002;96(4):429-33. Available from: DOI: 10.1016/So0359203(02)90383-4 PMID: 12497982

32. Conaty SJ, Dart S, Hayward AC, Lipman MC. Reasons for low reported treatment success in notified tuberculosis patients from a London hospital according to new outcome reporting. Commun Dis Public Health. 2004;7(1):73-6.PMID: 15137286

33. World Health Organization (WHO). Resolution WHA 61.17. Health of migrants. In: Sixty-first World Health Assembly, Geneva, 19-24 May 2008. Resolutions and decisions, annexes. Geneva: WHO; 2008:2 (document WHA61/2008/REC/1). Available from: http://apps.who.int/gb/ebwha/pdf_files/ WHA61-REC1/A61_REC1-en.pdf

34. Fernandes A, Miguel JP. Health and migration in European Union: better health for all in an inclusive society. 291173/09. Lisboa. Instituto Nacional de Saúde Doutor Ricardo Jorge 2009. Available from: http://www.insa.pt/sites/INSA/ Portugues/Publicacoes/Outros/Documents/Epidemiologia/ HealthMigrationEU2.pdf 